\title{
Dynamics of pollination and seed dispersal mutualisms at low density
}

$$
\text { Kayla R. S. Hale }{ }^{1 *} \text {, Daniel P. Maes }{ }^{1,2}, \text { \& Fernanda S. Valdovinos }{ }^{1,3^{*}}
$$

$3 \quad{ }^{1}$ Department of Ecology and Evolutionary Biology, University of Michigan, Biological

4 Sciences Building, 1105 North University Ave, Ann Arbor, MI 48109, USA.

$5 \quad{ }^{2}$ Department of Mathematics, University of Michigan, East Hall, 530 Church St, Ann

6 Arbor, MI 48109, USA.

$7{ }^{3}$ Department of Environmental Science and Policy, University of California, Davis,

8 Wickson Hall, One Shields Avenue, Davis, CA 95616.

9 *Corresponding authors; email: kaylasal@umich.edu, fvaldovinos@ucdavis.edu

10 Manuscript elements: Figure 1, Figure 2, Figure 3, Figure 4, Figure 5, Table 1, Online

11 Appendix A. Figures 1-4 are to print in color.

12 Keywords: transportation mutualism, reproductive services, population dynamics,

13 thresholds, Allee effects, germination, negative density-dependence

14 Manuscript type: Major Article.

15 Running title: Dynamics of mutualisms at low density.

16 Word count: 5564.

17 Acknowledgements: This research was supported by National Science Foundation Graduate

18 Research Fellowship DGE-1143953 to K.R.S.H., National Science Foundation Graduate

19 Research Fellowship DGE-1256260 to D.P.M., and National Science Foundation grant

20 DEB-1834497 to F.S.V.

21 Competing interests: The authors declare no competing interests. 
22 Statement of authorship: F.S.V. conceived the study; K.R.S.H. and D.P.M. analyzed the

23 models; K.R.S.H. and F.S.V. developed the biological interpretation of the model results;

24 K.R.S.H. wrote the manuscript with important contributions from F.S.V.; all authors

25 developed the models.

26 Code availability: Mathematica notebooks used to analyze the models are available by

27 request and will be available on GitHub upon acceptance. 
Abstract

30 Pollination and seed dispersal mutualisms are critical for biodiversity and ecosystem

31 services yet face mounting threats from anthropogenic perturbations that cause their

32 populations to decline. Characterizing the dynamics of these mutualisms when populations

33 are at low density is important to anticipate consequences of these perturbations. We

34 developed simple population dynamic models detailed enough to distinguish different

35 mechanisms by which plant populations benefit from animal pollination or seed dispersal.

36 We modeled benefits as functions of foraging rate by animals on plant rewards and

37 specified whether they affected plant seed set, germination, or negative density dependence

38 during recruitment. We found that pollination and seed dispersal mutualisms are stable at

39 high density but exhibit different dynamics at low density, depending on plant carrying

40 capacity, animal foraging efficiency, and whether populations are obligate upon their

41 partners for persistence. Under certain conditions, all mutualisms experience destabilizing

42 thresholds in which one population declines because its partner is too rare. Plants

43 additionally experience Allee effects when obligate upon pollinators. Finally, pollination

44 mutualisms can exhibit bistable coexistence at low or high density when plants are

45 facultative upon pollinators. Insights from our models can inform conservation efforts, as

46 mutualist populations continue to decline globally. 


\section{Introduction}

Pollination and seed dispersal mutualisms support vast amounts of biodiversity and

50 productivity in terrestrial ecosystems. Pollinators aid the reproduction of $78 \%$ and $94 \%$ of

51 flowering plants in temperate and tropical regions, respectively (Ollerton et al. 2011), while

52 seed dispersers aid the reproduction of $56 \%$ of plant species worldwide (Aslan et al. 2013).

53 In addition, the production of $75 \%$ of the world's major crops partially or completely

54 depend on pollinators (Klein et al. 2007). Unfortunately, these important mutualisms face

55 threats globally. A recent meta-analysis of plant regeneration in forests (Neuschultz et al.

56 2016) found that pollination and seed dispersal are the processes most vulnerable to human

57 disturbance including climate change, nutrient runoff, pesticide use, and invasive species

58 (Stachowicz 2001, Traveset \& Richardson 2006, Tylianakis et al. 2008, Zhou et al. 2013).

59 In fact, there is clear evidence showing the abundance of pollinators and key seed disperser

60 taxa such as frugivorous birds and mammals are declining globally (Potts et al. 2010, 2016,

61 Wotton \& Kelly 2011). Therefore, understanding the population dynamics of species

62 involved in these mutualisms, especially at low abundances, can inform predictions of the

63 consequences of those declines on the mutualisms, plant populations and crop production.

Historically, theoretical research (Gause \& Witt 1935, Vandermeer \& Boucher

1978, Addicott 1981, Wolin 1985, Bascompte et al. 2006, Okuyama \& Holland 2008,

66 Bastolla et al. 2009) used modified Lotka-Volterra type models (sensu Valdovinos 2019) to

67 investigate the dynamics of populations interacting mutualistically. This phenomenological

68 representation of mutualistic interactions as net positive effects between interacting species

69 provided insight for characterizing the effects of facultative, obligate, linear, and saturating 
70 mutualisms on the long-term stability of mutualistic systems. However, representing

71 mutualisms simply as positive effects between species overlooks important dynamics that

72 emerge from the specific mechanisms by which species positively affect each other. More

73 recent research advanced upon these phenomenological representations of mutualism by

74 developing models that account for consumer-resource mechanisms (Wright 1989, Holland

75 \& DeAngelis 2010, Valdovinos et al. 2013, Revilla 2015). Accounting for consumer-

76 resource mechanisms enabled the discovery of important dynamics, such Allee effects,

77 alternative states, transitions between mutualism and parasitism, competition among

78 species sharing mutualistic partners, and niche partitioning (May 1976, Soberón \&

79 Martinez del Rio 1981, Wells 1983, Wright 1989, Pierce \& Young 1986, Holland \&

80 DeAngelis 2010, Valdovinos et al. 2013, 2016, Revilla 2015, Valdovinos \& Marsland

812021 ) as well as the integration of these mutualisms into food web dynamics (Ringel et al.

82 1996, Hale et al. 2020). This research, however, mostly focused on animal dynamics (i.e.,

83 the consumer) and the dynamics of plant resources available to the animals (but see Wells

84 1983). To investigate the consequences of pollinator and seed disperser declines on plant

85 populations, more focus is needed on the mechanisms by which these animals affect plant

86 reproduction (Beckman et al. 2020). Here, we improve mechanistic understanding of the

87 ecological dynamics of these mutualisms by developing and analyzing consumer-resource

88 models that also account for simple mechanisms of reproductive benefits to plants.

Pollination and seed dispersal mutualisms share similarities as "transportation

90 mutualisms" (Bronstein 2015). In both cases, animals visit plants to feed upon rewards

91 (such as nectar and fruit) and provide reproductive services (such as transport of pollen and 
92 seeds) incidentally during foraging. However, these interactions differ in the mechanism of

93 reproductive benefit to plants. Pollinators increase plant seed set by facilitating cross-

94 fertilization, by moving pollen between conspecific plant individuals (Willmer 2011). Seed

95 dispersers increase plant recruitment by lessening density-dependent seed(ling) mortality

96 caused by predators, pathogens, and competitors and facilitating colonization of new

97 habitats (Wotton \& Kelly 2011, Moore \& Dittel 2020). Seed dispersal can also increase

98 germination by improving seed condition during passage through dispersers' guts (Fricke et

99 al. 2013). Here, we investigate the dynamical consequences of these various mechanisms of

100 reproductive benefits to plants. We find distinct dynamical behaviors at low population

101 densities, including thresholds, Allee effects, and bistable coexistence, depending on

102 species obligacy (i.e., their ability to persist without their partner), plant carrying capacity,

103 and animal foraging efficiency. We also show that pollination and seed dispersal

104 mutualisms have similar dynamics and stability at high density but differ in the ecological

105 conditions under which the populations are vulnerable to collapse.

106

108

109

110

111 versus seed dispersal interactions. We assume that the growth rate of animal and plant

112 populations are functions of the density-independent per-capita birth $\left(b_{A}, b_{P}\right)$ and death $\left(d_{A}\right.$, 
$113 d_{P}$ ) rates in absence of their mutualist, where subscripts $A$ and $P$ indicate animal and plant

114 populations, respectively. These population growth rates are also function of per-capita

115 self-limitation and other density-dependent processes $\left(s_{A}, s_{P}\right)$, and the benefits provided by

116 mutualists (see below). Our models are continuous in time, which accommodates species

117 with overlapping generations. They are also deterministic and ignore migration, which

118 allows us to focus on the dynamics that emerge from the mutualistic interactions,

119 unobscured by stochasticity and the dynamics of other patches. Table 1 summarizes our

120 parameter definitions for all models.

Population dynamics of animals

Pollinators and seed dispersers benefit from visiting plants by foraging for rewards

123 that offer primarily nutritional benefit (Willmer 2011, Jordano 2014). We therefore model

124 the change in animal population density $(\boldsymbol{A})$ over time $t$ as

$$
\frac{d \boldsymbol{A}}{d t}=b_{A} \boldsymbol{A}+\varepsilon C_{R}-s_{A} \boldsymbol{A}-d_{A} \boldsymbol{A}^{2}
$$

126 where the growth rate of the animal population increases proportionally to its consumption

127 rate of plant rewards, $C_{R}$. Parameter $\varepsilon$ is the efficiency of converting rewards to new animal

128 individuals via birth and maturation. We choose total consumption rate $C_{R}=\frac{a A \boldsymbol{P}}{1+a h \boldsymbol{P}}$,

129 following a Holling Type II functional response with attack rate $a$ and handling time $h$.

130 This encodes the reasonable assumption that animal consumption rate on plant rewards

131 saturates with increasing density of the plant population. Reproduction fueled by resources

132 other than plant rewards is included in $b_{A}$. We define an animal population $(\boldsymbol{A})$ as an

133 "obligate" mutualist of the plant population $(\boldsymbol{P})$ when $r_{A}=b_{A}-d_{A} \leq 0$, that is, when $\boldsymbol{A}$ 
134 cannot persist in the absence of its mutualist plant. Otherwise $\left(r_{A}>0\right), \boldsymbol{A}$ is "facultative"

135 and its population is self-sustaining.

136

138

140

141

142 151 dynamics of plants.

\section{Population dynamics of plants}

Plants benefit from reproductive services provided by animals while foraging. We define $b_{P}$, the plant birth rate, specifically as maximum per-capita seed set. We assume that negative density-dependence $\left(s_{P}\right)$ limits the seeds that survive and mature to reproductive adults, due to, e.g., seed competition during recruitment or the preferential attraction of natural enemies (Janzen-Connell effect). Additionally, we assume that plant reproduction is limited by the fraction of flowers that can be fertilized through wind pollination or selfing $(0 \leq f \leq 1)$ and the fraction of seeds that can germinate $(0 \leq g \leq 1)$ even when subjected to low negative density-dependence. Reproductive services from animals increase the density of mature (reproductive) plant individuals by pollinating flowers $(\varphi)$, improving germinability $(\gamma)$, or providing refuge from seed predation and other sources of densitydependent mortality during recruitment $(\sigma)$. Reproductive services are functions of animal visitation to plants, which we assume to be well approximated by the foraging rate on rewards $\left(C_{R}\right.$, Vázquez et al. 2005). Below, we derive separate models for pollination and seed dispersal based on the different mechanisms by which each affects the population

152 Pollination: Increase in realized seed set from pollinating flowers 
155 by animals can increase the fraction of plant flowers that are pollinated $(f)$ to a maximum

156 of $f+\varphi \leq 1$. We model animal-pollinated plant population dynamics $(\boldsymbol{P})$ as

$$
\frac{d \boldsymbol{P}}{d t}=\boldsymbol{P}\left[b_{P}\left(f+\varphi \frac{\delta_{P}}{1+\delta_{P}}\right) g-s_{P} \boldsymbol{P}-d_{P}\right]
$$

158 where benefit from pollination services $\left(\mathcal{S}_{P}\right)$ saturates as the fraction of flowers that are

159 pollinated approaches its maximum (also see Appendix A). We assume $\mathcal{S}_{P}=C_{R}$, where the

160 contribution of the animal population to per-plant seed set is the total foraging rate of

161 animals on plant rewards $\left(C_{R}\right)$. The direct dependence on plant density in this expression

162 accounts for the repeated interactions between plant and animal individuals required for

163 conspecific pollen transfer (Vázquez et al. 2005, Schupp et al. 2017). Using the Holling

164 Type II functional response for $C_{R}$ from above and simplifying the algebra yields our specific model for animal-pollination mutualisms:

166

$$
\left\{\begin{array}{c}
\frac{d \boldsymbol{P}}{d t}=\boldsymbol{P}\left[b_{P}\left(f+\varphi \frac{a \boldsymbol{A P}}{1+a h \boldsymbol{P}+a \boldsymbol{A P}}\right) g-s_{P} \boldsymbol{P}-d_{P}\right] \\
\frac{d \boldsymbol{A}}{d t}=\boldsymbol{A}\left[b_{A}+\varepsilon \frac{a \boldsymbol{P}}{1+a h \boldsymbol{P}}-s_{A} \boldsymbol{A}-d_{A}\right]
\end{array}\right.
$$

167 Plant benefits are limited both by the fraction of flowers available to pollinate $(\varphi)$ and by

168 pollinators' handling time on flowers $(h)$, with maximally effective reproductive services

169 leading the plant population to achieve close to its maximum per-capita seed set $\left(b_{P}\right)$.

170 Following our previous definitions, plant population $(\boldsymbol{P})$ is an obligate mutualist of $\boldsymbol{A}$ when

171 it cannot persist in its absence $\left(r_{P}=b_{P} f g-d_{P} \leq 0\right)$; otherwise, $\boldsymbol{P}$ is facultative $\left(r_{P}>0\right)$.

\section{Seed dispersal: Reduction in negative density-dependence or increase in germination}


Seed dispersers visit plants to forage on fruit, elaiosomes, or even seeds directly,

174 later depositing the seed away from the parent plant. This process increases recruitment by

175 reducing density-dependent mortality caused by predators or pathogens, which are most

176 abundant near adult plants (Wotton \& Kelly 2011, Fricke et al. 2013, Gómez et al. 2019,

177 Moore \& Dittel 2020). Seed dispersal may also increase germination by improving seed

178 condition during passage through dispersers' guts, which removes pathogens or provides

179 "chemical camouflage" from natural enemies (Fricke et al. 2013). We consider both cases

180 of seed dispersal services $\left(\mathcal{S}_{D}\right)$ below.

181 First, animals rescue seeds from predators, pathogens, and intraspecific competition

182 by transporting them away from parent plants. This can increase successful recruitment:

$$
\frac{d \boldsymbol{P}}{d t}=\boldsymbol{P}\left[b_{P} f g-\left(s_{P}-\sigma \frac{\mathcal{S}_{D}}{1+\mathcal{S}_{D}}\right) \boldsymbol{P}-d_{P}\right]
$$

184 where negative density-dependence $\left(s_{P}\right)$ can be reduced to a minimum of $s_{P}-\sigma \geq 0$ in the

185 presence of animals. Eqn. 4 implies that plants must be potentially persistent (have positive

186 demographic rates, $r_{P}=b_{P} f g-d_{P}>0$ ) to benefit from reductions to negative density-

187 dependence. Therefore, we only consider facultative plants $\left(r_{P}>0\right)$ for this case.

188 Second, animals may increase seed condition by masticating or digesting fruits.

189 This can improve germinability:

$$
\frac{d \boldsymbol{P}}{d t}=\boldsymbol{P}\left[b_{P} f\left(g+\gamma \frac{\delta_{D}}{1+\delta_{D}}\right)-s_{P} \boldsymbol{P}-d_{P}\right] .
$$


191 where the fraction of seeds that germinate $(g)$ can increase in the presence of animals to a

192 maximum of $g+\gamma \leq 1$. Plants may be obligate $\left(r_{P} \leq 0\right)$ or facultative $\left(r_{P}>0\right)$ upon their

193 animal partner for population persistence.

194 In both seed dispersal cases, reproductive services are directly related to

195 consumption rate of animals on plant rewards. We use $\mathcal{S}_{D}=C_{R} / \boldsymbol{P}$, where the per-plant

196 benefit of seed dispersal is proportional to the per-capita (i.e., per-plant) visitation rate of

197 animals on plants. This is because repeated interactions between plant and animal

198 individuals are possible but not required for effective seed dispersal, as they are for

199 effective pollination (Schupp et al. 2017). Using the Holling Type II functional response

200 from above yields our specific models for animal seed-dispersal mutualisms in which plants

201 benefit via escape from negative density-dependence:

202

$$
\left\{\begin{array}{c}
\frac{d \boldsymbol{P}}{d t}=\boldsymbol{P}\left[b_{P} f g-\left(s_{P}-\sigma \frac{a \boldsymbol{A}}{1+a h \boldsymbol{P}+a \boldsymbol{A}}\right) \boldsymbol{P}-d_{P}\right] \\
\frac{d \boldsymbol{A}}{d t}=\boldsymbol{A}\left[b_{A}+\varepsilon \frac{a \boldsymbol{P}}{1+a h \boldsymbol{P}}-s_{A} \boldsymbol{A}-d_{A}\right]
\end{array}\right.
$$

203 or via increased germination:

$$
\left\{\begin{array}{c}
\frac{d \boldsymbol{P}}{d t}=\boldsymbol{P}\left[b_{P} f\left(g+\gamma \frac{a \boldsymbol{A}}{1+a h \boldsymbol{P}+a \boldsymbol{A}}\right)-s_{P} \boldsymbol{P}-d_{P}\right] \\
\frac{d \boldsymbol{A}}{d t}=\boldsymbol{A}\left[b_{A}+\varepsilon \frac{a \boldsymbol{P}}{1+a h \boldsymbol{P}}-s_{A} \boldsymbol{A}-d_{A}\right]
\end{array}\right.
$$

205 In these specific forms, plant benefits are limited both by the fraction of seeds available to

206 germinate $(\gamma)$ or the negative density-dependent effects to reduce $(\sigma)$ and by dispersers'

207 handling time on rewards $(h)$.

\section{Costs of mutualism}


We do not model costs of mutualisms explicitly. We see three scenarios in which

210 our choice is justified ecologically. First, individual-level costs may be already accounted

211 for in the population-level parameters. For example, costs of producing nectar can lead to

212 fewer ovules in flowers (Pyke 1991, Brandenburg et al. 2012), which reduces the ovules

213 fertilized by animal visits and, therefore, can be accounted for by a reduced value of

214 parameter $\varphi$ in Eqn. 2. Second, individual-level costs may be on average (i.e., at the

215 population-level) of such a small effect over the lifespan of the individual that they can be

216 considered negligible. For example, nectar production is usually considered of low

217 energetic cost (Harder \& Barrett 1992, Revilla 2015). Third, other population-level

218 parameters may drown out the effect of costs from mutualism (Ford et al. 2015). For

219 example, nectar may be costly to produce for some plant species, but safe seed sites are

220 significantly more limiting to those plants' reproduction. Some systems may not conform to

221 these scenarios, in which case modeling costs could be important to understand their

222 dynamics.

\section{Analysis}

We performed phase plane analyses on our pollination (Eqn. 3) and seed dispersal

227 present results for the ecologically relevant cases where populations have positive densities

228 and can potentially coexist (i.e., are feasible). We investigated the behavior of populations

229 at low density, assessing our models for conditions under which Allee effects, thresholds,

230 and other alternative stable states occur. We use the term "Allee effects" for strong, 
231 demographic Allee effects, in which a population declines under a certain threshold of its

232 own density (Kramer et al. 2009). We define "threshold effects" as population declines

233 caused by the decline of its partner's density under a certain threshold (Vandermeer \&

234 Boucher 1978, Revilla 2015). Alternative stable states, such as single-species persistence or

235 bistable coexistence, occur when a system can settle stably into more than one equilibrium

236 depending only upon initial conditions ("historical accidents," May 1977).

\section{Results}

We found that pollination and seed dispersal mutualisms have similar dynamics and

239 stability at high density but differ in the ecological conditions under which the populations

240 are vulnerable to collapse. Different parameter regimes, in combination with whether each

241 partner is obligate or facultative, lead to different dynamics at low population densities.

242 Below, we describe these different dynamics for each of our models, assuming that

243 coexistence is feasible (but see Appendix A for mathematical details). Then, we describe

244 some ecological scenarios under which each outcome is likely to occur. We provide these

245 scenarios rather than providing formal mathematical conditions, because the latter are

246 complex and difficult to interpret due to the nonlinearities in our models.

Pollination

The shape and intersections of the plant and animal "nullclines" (curves of zero

249 change in population density) determine the dynamics of the system. Both species have

250 "trivial" nullclines at zero density. The non-trivial animal nullclines (black curves) are

251 concave down, increasing functions that saturate with respect to plant density (Figs. 1-3). 
252 The non-trivial animal-pollinated plant nullclines (green curves) are U-shaped when plants

253 are obligate mutualists (Fig. 1A-B) or increasing and concave up at high densities when

254 plants are facultative (Fig. 1C-G). Depending on the parameter regime, the facultative plant

255 nullcline may exhibit an inflection point at low densities in which it transitions from

256 concave down to up (e.g., Fig. 1D). Facultative animals and plants may persist in the

257 absence of their partners at density $K_{A}=r_{A} / s_{A}$ and $K_{P}=r_{P} / s_{P}$, respectively. These

258 densities are "carrying capacities" in the original sense of single-species equilibrium

259 densities (Vandermeer \& Boucher 1978). When coexistence occurs, it is at a higher density

260 than either species could achieve alone $\left(>K_{P},>K_{A}\right)$.

When plants are obligate (Fig. 1A-B), pollination mutualisms exhibit two non-

262 trivial equilibria: (1) A stable coexistence equilibrium (off-axes filled circle). Density past

263 this equilibrium in either species causes its population to decrease due to negative density-

264 dependence. Therefore, unbounded population growth cannot occur. (2) A saddle point that

265 attracts in one dimension but repels in the other (off-axes hollow circle), bisecting the plane

266 into two portions, as marked by the "separatrix" (dashed line). This separatrix marks out a

267 threshold under which obligate partners go extinct even if initially highly abundant

268 ("threshold effects," regions shaded light red). For example, following a trajectory from the

269 lower-right region in Fig. 1B, initially highly abundant obligate plants go extinct while

270 initially rare pollinators persist at $K_{A}$. These threshold effects occur because one species is

271 too low in density to provide sufficient benefits to its partner, causing the partner's

272 population to decline. The low-density species continues to benefit from mutualism but its

273 increase in density cannot occur fast enough to save the system from collapse. Above the 
274 separatrix, one or both species are of high enough density that benefits from mutualism

275 cause positive population growth in their partners and the system will achieve stable

276 coexistence.

Obligate animal-pollinated plants are additionally susceptible to Allee effects

278 (regions shaded dark red), where their population declines under a threshold of their own

279 density regardless of the density of their partner (Fig. 1A-B). This occurs because benefit is

280 proportional to the total consumption rate by animals, i.e., plants require obligate

281 outcrossing for successful pollination behavior. At very low plant density, even highly

282 abundant pollinators are limited by the time required to harvest and (incidentally) transfer

283 pollen between two plant individuals, and thus cannot provide pollination services at a rate

284 sufficient to allow plant population growth. Unsurprisingly, animals cannot acquire enough

285 food and decline either to extinction (Fig. 1A) or to $K_{A}$ (Fig. 1B) when plants are extinct.

286 When animal-pollinated plants are facultative (Fig. 1C-G), more diverse dynamics

287 are possible, depending on the relative growth rates of plants and animals and animals'

288 foraging efficiency. High-density stable coexistence always occurs if partners are originally

289 at high enough density. At lower density, threshold effects can occur when animals are

290 obligate mutualists (Fig.1C), but population growth from low densities is also possible (Fig.

291 1D-G) and even guaranteed when both partners are facultative mutualists (Fig. 1F-G).

292 Additionally, in a small parameter range, the mutualism can exhibit bistable coexistence

293 (Fig. 1D, 1F). Here, there are two possible points of stable coexistence which the system

294 will be attracted to - one at lower density and one at higher - based on initial conditions. A 
295 separatrix (dashed line) running through a central saddle point (hollow circle) marks out a

296 threshold under which the system will persist at the lower-density coexistence equilibrium.

By inspecting the intercepts of the nullclines with the axes, we gain intuition into

298 ecological scenarios when these different dynamics (threshold effects, stable coexistence,

299 or bistable coexistence) will occur. Threshold effects (Fig. 1C) can occur when

$300-\frac{r_{A}}{a\left(\varepsilon+h r_{A}\right)}>K_{P}$, that is, when the intercept of the animal nullcline with the $\mathrm{x}$-axis

301 (representing plant density) is greater than the intercept of the plant nullcline. This is most

302 likely when pollinators are highly obligate upon plants $\left(r_{A} \ll 0\right)$ and when plants have low

303 carrying capacity, $K_{P}$. That is, when the environment is hostile due to, for example, high

304 density-independent mortality $\left(d_{P}, d_{A}\right)$ or high negative density-dependence for plants $\left(s_{P}\right)$.

305 On the other hand, growth from low density (Fig. 1D-G) can occur under the opposite

306 condition, when $-\frac{r_{A}}{a\left(\varepsilon+h r_{A}\right)} \leq K_{P}$. That is, when the intercept of the animal nullcline with

307 the $\mathrm{x}$-axis is less than the intercept of the plant nullcline. This is most likely when animals

308 are facultative or only weakly obligate and plants have high $K_{P}$. However, if this condition

309 holds but plants have low $K_{P}$, bistable coexistence may occur. Bistable coexistence is most

310 likely when pollinators have near-zero population growth rate $\left(r_{A}\right)$ in the absence of plants,

311 regardless of whether they are obligate or facultative. Additionally, the potential for

312 bistable coexistence is strongly modulated by pollinators' foraging efficiency, especially

313 their attack rate $(a)$ or search time $(1 / a)$ for floral rewards. Within a narrow range of $a$,

314 bistable coexistence can occur (Fig. 4A). At higher $a$, only a single, high-density, 
315 coexistence equilibrium is present. At lower $a$, stable coexistence occurs only at low

316 density or is even infeasible.

Our seed dispersal models (Figs. 2-3) display simpler dynamics than our pollination

319 model. Seed dispersers follow the same dynamics as pollinators (black curves). The non-

320 trivial nullclines for seed-dispersed plants are concave up, increasing functions (green

321 curves), bounded by a vertical asymptote on the right. This results in stable coexistence at

322 higher density than either species could achieve alone with the potential for threshold

323 effects, but not the bistable coexistence or Allee effects dynamics described for pollination

324 mutualisms.

When seed dispersers benefit plants through reduced negative density-dependence

326 (Fig. 2), plants are always facultative (see Methods) and can persist in the absence of

327 animal mutualists at density $K_{P}$. The same qualitative conditions hold as described for the

328 pollination model as to whether the system exhibits threshold effects $\left(-\frac{r_{A}}{a\left(\varepsilon+h r_{A}\right)}>K_{P}\right.$, Fig.

329 2A) or a single stable coexistence equilibrium $\left(-\frac{r_{A}}{a\left(\varepsilon+h r_{A}\right)} \leq K_{P}\right.$, Fig. 2B) when animals are 330 obligate mutualists.

When seed dispersers benefit plants through increased seed germination (Fig. 3),

332 plants may be obligate or facultative, with population collapse or persistence at $K_{P}$,

333 respectively, in the absence of animals. When at least one partner is an obligate mutualist,

334 threshold effects can always occur, leading to extinction if at least one partner is at low 
335 enough density (Fig. 3A-B, 3D). As shown for the previous models, threshold effects can

336 occur when $-\frac{r_{A}}{a\left(\varepsilon+h r_{A}\right)}>K_{P}$ (Fig. 3A, 3D). They can also occur in this model when

$337-\frac{r_{P}}{a r_{P_{\max }}}>K_{A}$, where $r_{P_{\max }}=r_{P}+b_{P} f \gamma$. That is, when the intercept of the plant nullcline

338 with the $y$-axis (representing animal density) is greater than the intercept of the animal

339 nullcline (Fig. 3A-B).

340 In both seed dispersal cases, threshold effects are most likely when one partner is

341 highly obligate $\left(r_{i} \ll 0\right)$ and when plants are difficult, but not impossible, for dispersers to

342 find on the landscape (low search time, 1/a). At low $a$, threshold effects occur because

343 animals can never achieve a high enough foraging rate to prevent both species from decline

344 below the threshold density, unless they are initialized above the threshold density (Fig.

345 4B-C). At even lower $a$, coexistence is infeasible while at higher $a$, dispersers are adept at

346 finding plants, and both populations grow quickly to high density stable coexistence.

347 However, growth from low density with ultimate stable coexistence at high density is also

348 possible if neither partner is highly obligate and dispersers are efficient at finding plants

349 (Fig. 3C, 3E). Indeed, it is the only outcome when both partners are facultative (Fig. 2C, $350 \quad 3 F)$.

Discussion

Pollination and seed dispersal mutualisms support the reproduction of a vast number

353 of plant species globally, including crops (Klein et al. 2007, Aslan et al. 2013, Garibaldi et 
356 (Tylianakis et al. 2008, Traveset \& Richardson 2006, 2014, Potts et al. 2010, 2016,

357 Beckman et al. 2020). These potential disruptions increase the need to study the dynamics

358 of mutualisms when the interacting populations exhibit low density. Theoretical studies

359 have traditionally investigated these dynamics at low density by using Lotka-Volterra

360 models. Our work builds from those studies by developing novel consumer-resource

361 models of mutualistic interactions (sensu Holland \& DeAngelis 2010) that incorporate

362 simple mechanisms of reproductive benefits to plant populations. Our results expand the

363 understanding of pollination and seed dispersal mutualisms by elucidating dynamical

364 consequences of mechanisms by which pollinators and seed dispersers benefit plant

365 reproduction when visiting them as consumers.

366 We found threshold effects, Allee effects, and bistable coexistence in our models,

367 with transitions between these dynamics affected by partners' obligacy, environmental

368 hostility, and animals' foraging efficiency on plant rewards (Fig. 5). Threshold effects

369 occur in both pollination and seed dispersal mutualisms when at least one mutualist is

370 obligate because low-density populations cannot provide enough benefit to rescue their

371 higher density partner from decline when animal foraging efficiency on plant rewards is

372 low (Figs. 1A-C, 2A, 3A-B, 3D, 4B-C). Allee effects occur in obligate animal-pollinated

373 plants because the plants can become too low in density to attract sufficient pollinator visits

374 to save their own population (Figs. 1A-B). Bistable coexistence occurs in pollination

375 mutualisms when plants are facultative but the environment is hostile for plants (low

376 carrying capacity) and animals are of intermediate foraging efficiency (Figs. 1D, 1F, 4A).

377 Conversely, when the environment is benign (high plant carrying capacity) and animals are 
378 efficient foragers (Fig. 4A, 4C), populations grow from low density with subsequent stable

379 coexistence at a single, high-density equilibrium (Figs. 1E, 1G, 2B, 3C, 3E). This single,

380 high-density equilibrium is guaranteed for seed dispersal mutualisms when both partners

381 are facultative (Fig. 2C, 3F).

382 Threshold effects, population declines due to rarity of a mutualistic partner, were

383 first described in the late 1970s for obligate mutualists that strongly benefit each other

384 (May 1976, Vandermeer \& Boucher 1978). The strong mutualistic interaction allowed the

385 species to persist at high densities but was not sufficient for coexistence at densities below

386 the threshold. This research used the Lotka-Volterra model for mutualism with positive

387 interacting coefficients and linear functional responses, but the same qualitative results at

388 low density are found when saturation is added to the functional responses (Vandermeer \&

389 Goldberg 2013). Indeed, models incorporating consumer-resource mechanisms also predict

390 these thresholds in density, below which the system collapses (May 1976, Soberón \&

391 Martinez del Rio 1981, Wells 1983, Wright 1989, Fishman \& Hadany 2010, Revilla 2015).

392 In contrast, a model for "unidirectional" consumer-resource mutualisms, which include

393 both animal-pollination and seed dispersal interactions, predicts thresholds in density above

394 which the system collapses (Holland \& DeAngelis 2010). The collapse occurs due to

395 animal overexploitation of plant rewards.

396 Despite their ubiquity in theoretical work (Hale \& Valdovinos 2021), threshold

397 effects have been difficult to observe empirically (Latty \& Dakos 2019, Hillebrand et al.

398 2020). Our work suggests that threshold effects would only be observed in pollination

399 systems when pollinators are obligate and plants are facultative but in a hostile environment 
400 (low carrying capacity, Figs. 1C, 5). Otherwise, destabilization of the system would be

401 attributed to Allee effects in plants (Figs. 1A-B, 5). Threshold effects may be easier to

402 observe in seed dispersal systems because they would manifest when either partner's

403 density drops below the critical threshold (Fig. 2A, 3A-C). As a potential example, Wotton

404 and Kelly (2011) observed that seed survival of two species of New Zealand trees dropped

405 dramatically when fruit consumption by dispersers crossed below $30 \%$.

406

Allee effects may also cause irrecoverable declines in populations at low density.

407 We use "Allee effects" to describe self-induced, as opposed to partner-induced, declines at

408 low density. We find Allee effects in plant populations that are obligate mutualists of

409 pollinators, due to, e.g., high self-incompatibility. This result is consistent with pollination

410 ecology because pollinators' benefits to plants tend to decrease at low plant density due to

411 increased search time, leading to a decrease in visitation rate and pollen limitation (Forsyth

412 2003). Low plant density may also decrease visitation rate due to insufficient floral displays

413 or reduce visit quality due to pollen dilution (Forsyth 2003). Indeed, pollinator-mediated

414 Allee effects have been observed empirically in highly self-incompatible plant species

415 (Kramer et al. 2009). We show that reductions in visit quantity alone are sufficient to

416 induce an Allee effect, whereas natural populations likely experience both a decrease in

417 visit quality and quantity at low plant density. In contrast, we did not find Allee effects in

418 our seed dispersal models. This is consistent with the mechanisms by which seed dispersers

419 benefit plants. Seed dispersers benefit plants by increasing the seed germination or their

420 survival to adulthood through refuge from intraspecific competition and natural enemies

421 (seed predators, herbivores) that are attracted to regions of high food densities (i.e., the 
422 Janzen-Connell effect, Janzen 1970, Connell 1971, Wotton \& Kelly 2011, Fricke et al.

423 2013, Moore \& Dittel 2020). These plants are thus less likely to experience Allee effects,

424 because at low density the primary threats are similarly reduced.

425 Bistable coexistence occurs when partners coexist stably either at low or high

426 density, depending upon if populations dip below a certain threshold of density. It is, to our

427 knowledge, a novel result in mutualism models (but see Zhang 2003 and Holland \&

428 DeAngelis 2010 for examples when mutualism can transition dynamically to competition

429 or parasitism interactions). We observed bistable coexistence in pollination mutualisms

430 because the dependence on plant density due to the required transfer of pollen between

431 conspecific plants causes an inflection in the plant nullcline when facultative (Fig. 1D, 1F).

432 At intermediate foraging efficiency, pollinators can find plants, but do so inefficiently

433 enough (low enough attack rate) that the plant and animal populations never achieve a high

434 enough growth rate to escape a low-density stable attractor, where plants stay relatively rare

435 and animals stay inefficient at finding them. This happens unless initial plant abundance is

436 high enough to be easily found by animals so that sufficient mutualistic benefits can be

437 exchanged to allow stable coexistence at high density. This type of bistability may occur in

438 pollination systems where plant abundance explains the variability of total benefits that

439 plant populations receive from pollinator visits (Vázquez et al. 2007), with higher total

440 benefits received by more abundant plants that can sustain highly abundant pollinators, and

441 lower total benefits received by rare plants that would only sustain low pollinator

442 abundance. 
The influence of environmental hostility or stress has received much attention as a

444 potential cause for tipping points (Beisner et al. 2003, Lever et al. 2014, Kéfi et al. 2016,

445 Latty \& Dakos 2019, Hillebrand et al. 2020, Huang \& D’Odorico 2020), which we also

446 find here (Fig. 5). Species may transition from "facultative" to "obligate" mutualists with

447 increasing death rate $\left(d_{i}\right)$, leading to increased vulnerability in terms of the potential to

448 experience low-density thresholds. Additionally, lower carrying capacity $\left(K_{i}=r_{i} / s_{i}\right)$ due

449 to high negative density-dependence $\left(s_{i}\right)$, may cause a species to exhibit only low-density

450 or infeasible coexistence. Here, we highlight the effect of foraging efficiency on critical

451 transitions between dynamics (Figs. 4-5). Crypticity of rewards due to habitat

452 fragmentation, poor attack rate due to mismatched traits, or low preference due to declines

453 in rewards quality may shift animal foraging efficiency, may cause a previously stable

454 system at high density to shift to bistability, threshold effects or, in the worst case, collapse

455 to infeasible coexistence. Similarly, Valdovinos and Marsland (2021) identified the quality

456 of visits needed from pollinators for plants to persist. Below such threshold, the plant

457 species and the animals depending on those plants go extinct. This suggests that the

458 parameter of foraging efficiency (analogous to visit quality in their model) could be an

459 important topic for future investigation.

$460 \quad$ Overall, we find that obligate mutualisms are most at risk for experiencing

461 destabilizing thresholds, which may have cascading effects on the rest of the ecological

462 community (Latty \& Dakos 2019). The existence of thresholds suggests that environmental

463 change could shift a mutualistic system from above a separatrix to below it. This will lead

464 to collapse of the system under certain conditions, but in very special cases, the system may 
465 instead shift to a lower stable coexistence equilibrium, and still exhibit positive growth at

466 low density. Though this is a preferable outcome to deterministic extinction, these

467 dynamics still allow the system to reach very low population densities, and thus to be more

468 vulnerable to environmental or demographic stochasticity. However, the highly stable

469 outcomes for facultative mutualists, and particularly for facultative plants with high

470 carrying capacity, suggest that these species may buffer communities from perturbations to

471 low density in a network setting (also see van der Heide et al. 2020). This is because

472 mutualistic networks are typically highly nested, which means that generalist species tend

473 to interact with both generalist and specialist species but specialist species with only

474 generalists (Bascompte et al. 2003). Then, a nested network with generalist plants (by

475 definition facultative to each of their animal mutualists), that also have sufficiently high

476 carrying capacity, may not suffer threshold effects caused by rare, obligate animal

477 populations. In other words, those generalist plants with high carrying capacity may make

478 the network robust to potential threshold effects.

A unique aspect of our modelling approach is that we specify whether plants benefit

480 according to animal total or per-capita visitation rate. Our choice for pollination benefits to

481 plants to scale with animal total visitation rate accounts for outcrossing (Vázquez et al.

482 2005). This, to the best of our knowledge, makes our pollination model unique in the

483 literature of two-species models (but see Valdovinos et al. 2013, 2016, Hale et al. 2020 for

484 network models). In addition, visitation in our models differs from previous work (e.g.,

485 Revilla 2015) because we use nonlinear (specifically, Holling Type II) saturating functional

486 responses for consumption rate. This allows both plants and animals to experience intra- 
487 specific competition for benefits, set by the maximum time animals can spend foraging on

488 rewards. Lastly, we impose a direct limitation to plant benefits, so that they saturate due to

489 limited ovules or seeds. In this way, plant benefits in our seed dispersal models follow the

490 Beddington-DeAngelis functional response, which was formulated for consumers

491 experiencing exploitation competition.

Our models can also accommodate functional responses interpreted as "net benefit"

493 curves (e.g., Holland et al. 2002, Morris et al. 2010) if costs and benefits affect the same

494 vital rate. That is, if "costs" simply reduce the benefits that accrue to a given vital rate (e.g.,

495 Brandenburg et al. 2012). Under this interpretation, unimodal functional forms may arise

496 (e.g., Morris et al. 2010) which could lead to substantially different dynamical predictions

497 than those presented here. Finally, our models, as well as previous ones, assume that

498 mutualisms have population-level impacts. Most empirical studies, however, quantify the

499 benefits and costs of mutualisms at the individual level in terms of fitness or even by using

500 a single proxy for fitness (Bronstein 2001, Ford et al. 2015). Those effects do not

501 necessarily imply population- or community-level impacts of mutualism (Flatt \& Weisser

502 2000, Ford et al. 2015). Therefore, empirical work on population dynamics of mutualisms

503 is of foremost importance to evaluate historical and current ecological theory on

504 mutualisms.

\section{Conclusion}

This research increased mechanistic understanding of the dynamics of plant-animal

507 mutualisms by analyzing consumer-resource models that incorporate simple mechanisms of 
508 reproductive benefits to plants. We found that these mutualisms may be vulnerable to

509 declines at low density due to Allee and threshold effects and identified potential bistable

510 coexistence. We also characterized the ecological scenarios under which those dynamical

511 behaviors are most likely to occur, which has been recognized a conservation priority

512 (Latty \& Dakos 2019). Future work should continue to investigate how different

513 mechanisms of mutualism manifest in different population dynamics at low density, both to

514 advance ecological understanding and to aid in conservation objectives.

\section{References}

516 Addicott, J. F. 1981. Stability properties of 2-species models of mutualism: Simulation

517 studies. Oecologia 49:42-49.

518 Aslan, C. E., E. S. Zavaleta, B. Tershy, and D. Croll. 2013. Mutualism disruption Threatens

519 global plant biodiversity: A Systematic Review. PloS ONE 8.

520 Bascompte, J., Jordano, P., Melian, C.J. and Olesen, J.M. 2003. The nested assembly of

521 plant-animal mutualistic networks. Proceedings of the National Academy of Sciences USA

$522 \quad 100: 9383-9387$.

523 Bascompte, J., P. Jordano, and J. M. Olesen. 2006. Asymmetric coevolutionary networks

524 facilitate biodiversity maintenance. Science 1:3-5.

525 Bastolla, U., M. A. Fortuna, A. Pascual-García, A. Ferrera, B. Luque, and J. Bascompte.

526 2009. The architecture of mutualistic networks minimizes competition and increases

527 biodiversity. Nature 458:1018-1020. 
528 Beckman, N. G., C. E. Aslan, H. S. Rogers, O. Kogan, J. L. Bronstein, J. M. Bullock, F.

529 Hartig, et al. 2020. Advancing an interdisciplinary framework to study seed dispersal

530 ecology. AoB PLANTS 12:1-18.

531 Beisner, B. E., D. T. Haydon, and K. Cuddington. 2003. Alternative stable states in

532 ecology. Frontiers in Ecology and the Environment 1:376-382.

533 Brandenburg, A., C. Kuhlemeier, and R. Bshary. 2012. Hawkmoth pollinators decrease

534 seed set of a low-nectar Petunia axillaris line through reduced probing time. Current

535 Biology 22:1635-1639.

536 Bronstein, J. L. 2001. The costs of mutualism. American Zoologist 41:825-839.

537 Bronstein, J. L. 2015. The study of mutualism. Pages 3-19 in J. L. Bronstein, ed.

538 Mutualism. Oxford University Press, Oxford.

539 Connell, J. H. 1971 On the role ofnatural enemies in pre- venting competitive exclusion in

540 some marine mammals and forest trees. In Dynamics of populations (eds P. J. den Boer \&

541 G. R. Gradwell), pp. 298-312. Wageningen, The Netherlands: Centre for Agricultural

542 Publishing and Documentation.

543 Courchamp, F., L. Berec, and J. Gascoigne 2008. Population dynamics: modelling

544 demographic Allee effects. In Allee Effects in Ecology and Conservation, Oxford

545 University Press, Oxford.

546 Edwards, W., M. Dunlop, and L. Rodgerson. 2006. The evolution of rewards: Seed

547 dispersal, seed size and elaiosome size. Journal of Ecology 94:687-694. 
548 Fishman, M. A., and L. Hadany. 2010. Plant-pollinator population dynamics. Theoretical

549 Population Biology 78:270-277.

550 Flatt, T., and W. W. Weisser. 2000. The effects of mutualistic ants on aphid life. Ecology

$55181: 3522-3529$.

552 Ford, K. R., J. H. Ness, J. L. Bronstein, and W. F. Morris. 2015. The demographic

553 consequences of mutualism: ants increase host-plant fruit production but not population

554 growth. Oecologia 179:435-446.

555 Forsyth, S. A. 2003. Density-dependent seed set in the Haleakala silversword: Evidence for 556 an Allee effect. Oecologia 136:551-557.

557 Fricke, E. C., M. J. Simon, K. M. Reagan, D. J. Levey, J. A. Riffell, T. A. Carlo, and J. J.

558 Tewksbury. 2013. When condition trumps location: Seed consumption by fruit-eating birds

559 removes pathogens and predator attractants. Ecology Letters 16:1031-1036.

560 Garibaldi, L. A., I. Steffan-Dewenter, R. Winfree, M. A. Aizen, R. Bommarco, S. A.

561 Cunningham, C. Kremen, et al. 2013. Wild Pollinators Enhance Fruit Set of Crops

562 Regardless of Honey Bee Abundance. Science 339:1608-1611.

563 Gause, G. F., and A. A. Witt. 1935. Behavior of mixed populations and the problem of

564 natural selection. American Naturalist 69:596-609.

565 Gómez, J. M., E. W. Schupp, and P. Jordano. 2019. Synzoochory: the ecological and

566 evolutionary relevance of a dual interaction. Biological Reviews 94:874-902. 
567 Hale, K. R. S., F. S. Valdovinos, and N. D. Martinez. 2020. Mutualism increases diversity,

568 stability, and function of multiplex networks that integrate pollinators into food webs.

$569 \quad$ Nature Communications 11.

570 Harder, L. D., and S. C. H. Barrett. 2008. The Energy Cost of Bee Pollination for

571 Pontederia cordata (Pontederiaceae). Functional Ecology 6:226-233.

572 Hillebrand, H., I. Donohue, W. S. Harpole, D. Hodapp, M. Kucera, A. M. Lewandowska, J.

573 Merder, et al. 2020. Thresholds for ecological responses to global change do not emerge

574 from empirical data. Nature Ecology and Evolution 4:1502-1509.

575 Holland, J. N., and D. L. DeAngelis. 2010. A consumer-resource approach to the density-

576 dependent population dynamics of mutualism. Ecology 91:1286-1295.

577 Holland, J. N., D. L. DeAngelis, and J. L. Bronstein. 2002. Population dynamics and

578 mutualism: Functional responses of benefits and costs. American Naturalist 159:231-244.

579 Holland, J. N., Okuyama, T., \& DeAngelis, D. L. 2006. Comment on “Asymmetric

580 coevolutionary networks facilitate biodiversity maintenance." Science, 313:1887-1887.

581 Huang, H., and P. D’Odorico. 2020. Critical Transitions in Plant-Pollinator Systems

582 Induced by Positive Inbreeding-Reward-Pollinator Feedbacks. iScience 23:100819.

583 Janzen, D. H. 1970. Herbivores and number of tree species in tropical forests. American

584 Naturalist 104:501-508. 
585 Jordano P. 2014. Fruits and frugivory. Pages 125-166 in R. S. Gallagher, ed. Seeds: The 586 ecology of regeneration in plant communities. CABI, Boston.

587 Kéfi, S., M. Holmgren, and M. Scheffer. 2016. When can positive interactions cause 588 alternative stable states in ecosystems? Functional Ecology 30:88-97.

589 Klein, A. M., B. E. Vaissière, J. H. Cane, I. Steffan-Dewenter, S. A. Cunningham, C. 590 Kremen, and T. Tscharntke. 2007. Importance of pollinators in changing landscapes for 591 world crops. Proceedings of the Royal Society B: Biological Sciences 274:303-313.

592 Kramer, A. M., B. Dennis, A. M. Liebhold, and J. M. Drake. 2009. The evidence for Allee 593 effects. Population Ecology 51:341-354.

594 Latty, T., and V. Dakos. 2019. The risk of threshold responses, tipping points, and 595 cascading failures in pollination systems. Biodiversity and Conservation 28:3389-3406.

596 Lever, J. J., E. H. van Nes, M. Scheffer, and J. Bascompte. 2014. The sudden collapse of 597 pollinator communities. Ecology Letters 17:350-359.

598 May, R. M. 1976. Theoretical Ecology, Principles and Applications. Blackwell Scientific, 599 Oxford.

600 May, R. M. 1977. Thresholds and breakpoints in ecosystems with a multiplicity of stable 601 states. Nature 269:471-477.

602 Moore, C. M., and J. W. Dittel. 2020. On mutualism, models, and masting: The effects of 603 seed-dispersing animals on the plants they disperse. Journal of Ecology 108:1775-1783. 
604 Morris, W. F., D. P. Vázquez, and N. P. Chacoff. 2010. Benefit and cost curves for typical

605 pollination mutualisms. Ecology 91:1276-1285.

606 Neuschulz, E. L., T. Mueller, M. Schleuning, and K. Böhning-Gaese. 2016. Pollination and

607 seed dispersal are the most threatened processes of plant regeneration. Scientific Reports

$608 \quad 6: 6-11$.

609 Okuyama, T., and J. N. Holland. 2008. Network structural properties mediate the stability

610 of mutualistic communities. Ecology Letters 11:208-216.

611 Ollerton, J., R. Winfree, and S. Tarrant. 2011. How many flowering plants are pollinated by

612 animals? Oikos 120:321-326.

613 Pierce, N. E., and W. R. Young. 1986. Lycaenid butterflies and ants: Two-species stable

614 equilibria in mutualistic, commensal, and parasitic Interactions. American Naturalist

$615 \quad 128: 216-227$.

616 Potts, S. G., J. C. Biesmeijer, C. Kremen, P. Neumann, O. Schweiger, and W. E. Kunin.

617 2010. Global pollinator declines: Trends, impacts and drivers. Trends in Ecology and

618 Evolution 25:345-353.

619 Potts, S. G., V. Imperatriz-Fonseca, H. T. Ngo, M. A. Aizen, J. C. Biesmeijer, T. D.

620 Breeze, L. v. Dicks, et al. 2016. Safeguarding pollinators and their values to human well-

621 being. Nature 540:220-229.

622 Pyke, G. H. 1991. What does it cost a plant to produce floral nectar? Nature 350:58-59. 
623 Revilla, T. A. 2015. Numerical responses in resource-based mutualisms: A time scale

624 approach. Journal of Theoretical Biology 378:39-46.

625 Ringel, M. S., H. H. Hu, and G. Anderson. 1996. The stability and persistence of

626 mutualisms embedded in community interactions. Theoretical Population Biology 50:281-

627297.

628 Schupp, E. W., P. Jordano, and J. M. Gómez. 2017. A general framework for effectiveness

629 concepts in mutualisms. Ecology Letters 20:577-590.

630 Soberón, J. M., and C. Martinez del Rio. 1981. The dynamics of a plant-pollinator

631 interaction. Journal of Theoretical Biology 91:363-378.

632 Stachowicz, J. J. 2001. The structure of ecological communities. BioScience 51:235-246.

633 Traveset, A. and Richardson, D. M. 2006. Biological invasions as disruptors of plant

634 reproductive mutualisms. Trends in Ecology and Evolution 21:208-216.

635 Traveset, A. and Richardson, D. M. 2014. Mutualistic interactions and biological

636 invasions. Annual Review of Ecology, Evolution, and Systematics 45:89-113.

637 Tylianakis, J. M., R. K. Didham, J. Bascompte, and D. A. Wardle. 2008. Global change and

638 species interactions in terrestrial ecosystems. Ecology Letters 11:1351-1363.

639 Valdovinos, F. S. 2019. Mutualistic networks: moving closer to a predictive theory.

640 Ecology Letters 22:1517-1534. 
641 Valdovinos, F.S., Moisset de Espanes, P., Flores, J.D. \& Ramos-Jiliberto, R. 2013.

642 Adaptive foraging allows the maintenance of biodiversity of pollination networks. Oikos

$643 \quad 122: 907-917$.

644 Valdovinos, F. S., B. J. Brosi, H. M. Briggs, P. Moisset de Espanés, R. Ramos-Jiliberto, 645 and N. D. Martinez. 2016. Niche partitioning due to adaptive foraging reverses effects of 646 nestedness and connectance on pollination network stability. Ecology Letters 19:12776471286.

648 Valdovinos, F.S., and Marsland, R. 2021. Niche theory for mutualism: A graphical

649 approach to plant-pollinator network dynamics. American Naturalist 197:393-404.

650 van der Heide, T., C. Angelini, J. de Fouw, and J. S. Eklöf. 2020. Facultative mutualisms:

651 A double-edged sword for foundation species in the face of anthropogenic global change.

652 Ecology and Evolution 11:29-44.

653 Vandermeer, J. H., and D. H. Boucher. 1978. Varieties of mutualistic interaction in 654 population models. Journal of Theoretical Biology 74:549-558.

655 Vandermeer, J. H., and Goldberg, D. E. 2013. Pages 225-238 in Population ecology: First 656 principles. Princeton University Press, Princeton.

657 Vázquez, D. P., W. F. Morris, and P. Jordano. 2005. Interaction frequency as a surrogate 658 for the total effect of animal mutualists on plants. Ecology Letters 8:1088-1094. 
659 Vázquez, D. P., C. J. Melián, N. M. Williams, N. Blüthgen, B. R. Krasnov, and R. Poulin.

660 2007. Species abundance and asymmetric interaction strength in ecological networks.

661 Oikos 116:1120-1127.

662 Wells, H. 1983. Population equilibria and stability in plant-animal pollination systems.

663 Journal of Theoretical Biology 100:685-699.

664 Willmer, P. 2011. Pollination and Floral Ecology. Princeton University Press, Princeton.

665 Wolin, C. L., and L. R. Lawlor. 1984. Models of facultative mutualism: Density effects.

666 American Naturalist 124:843-862.

667 Wolin, C.L. 1985. The population dynamics of mutualistic systems. Pages in 248-269. D.

668 H. Boucher, ed. The Biology of Mutualism. Oxford University Press, Oxford.

669 Wotton, D. M., and D. Kelly. 2011. Frugivore loss limits recruitment of large-seeded trees.

670 Proceedings of the Royal Society B: Biological Sciences 278:3345-3354.

671 Wright, D. H. 1989. A simple, stable model of mutualism incorporating handling time.

672 American Naturalist 134:664-667.

673 Zhang, Z. 2003. Mutualism or cooperation among competitors promotes coexistence and

674 competitive ability. Ecological Modelling 164:271-282.

675 Zhou, Y., C. Newman, J. Chen, Z. Xie, and D. W. Macdonald. 2013. Anomalous, extreme

676 weather disrupts obligate seed dispersal mutualism: Snow in a subtropical forest ecosystem.

677 Global Change Biology 19:2867-2877. 

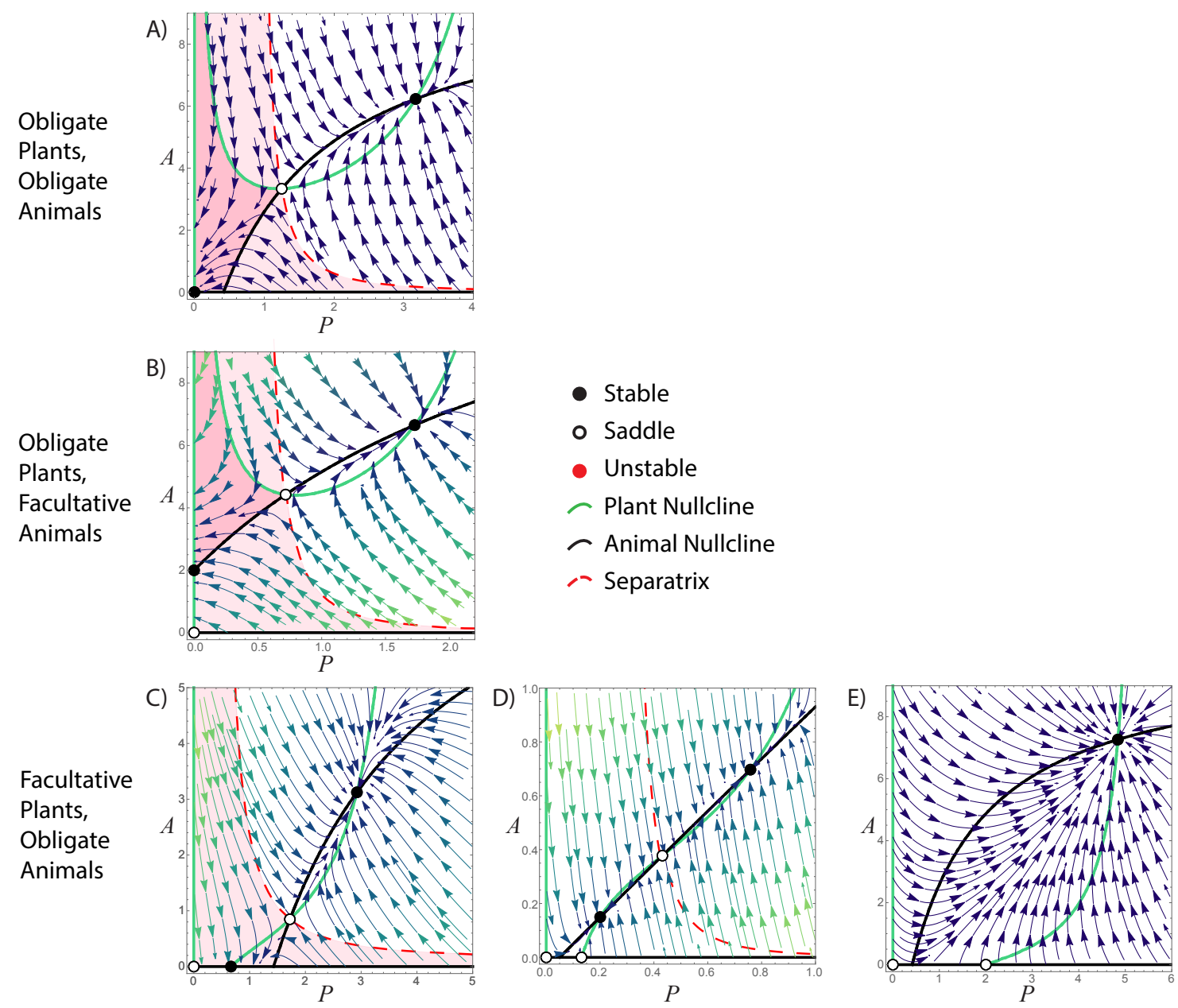

Facultative

Plants,

Facultative

Animals
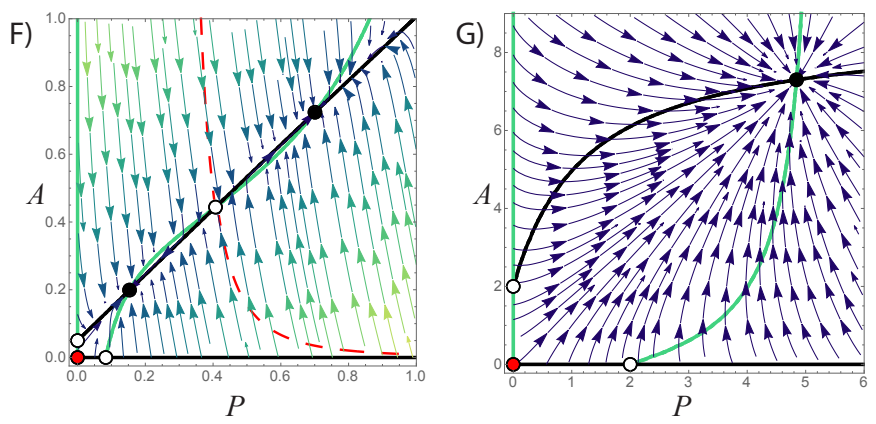

679 Figure 1. Phase plane diagrams for plant-pollinator mutualisms. Nullclines are curves

680 of zero growth for plant (green) or animal pollinator (black) populations. Equilibria (dots)

681 occur at the intersection of plant and animal nullclines. Filled black equilibria are stable

682 attractors, filled red equilibria are unstable repellers, and hollow equilibria are saddle 
683 points, which repel in one dimension and attract in the other. Arrows show the directions of

684 population change for plants (x-axis) and animals (y-axis), with lighter colors indicating a

685 faster rate of change. Dynamics depend upon the parameterization of the model and

686 whether plants and animals are obligate or facultative mutualists (rows). When plants are

687 obligate (A, B), they experience Allee effects at low density (dark red shaded region).

688 Plants cannot attract sufficient pollinator visitation to support their own population growth,

689 leading to collapse. When either plants or animals are obligate, both partners experience

690 threshold effects (light red shaded region). Below the threshold marked by the separatrix

691 (dashed line), one population's density is too low to support its partner's growth, resulting

692 in system collapse to extinction (A), animal-only persistence at $K_{A}=\left(b_{A}-d_{A}\right) / s_{A}(\mathbf{B})$, or

693 plant-only persistence at $K_{P}=\left(b_{P} f g-d_{P}\right) / s_{P}(\mathbf{D})$. Thus, depending upon the initial

694 densities of both partners, the mutualism may persist stably or collapse. When one partner

695 is facultative, stable coexistence may be the only outcome $(\mathbf{C}, \mathbf{E}, \mathbf{G})$. However, in a small

696 parameter space, bistable coexistence may occur $(\mathbf{D}, \mathbf{F})$. Here, the separatrix divides the

697 region in which the system will be attracted to the low- or high-density stable coexistence

698 equilibrium, depending upon whether initial densities are below or above the separatrix,

699 respectively. Parameter values are fixed to the following: $b_{P}=1, f=1, \varphi=0.5, g=$

$7001, s_{P}=0.15, a=0.8, h=1, b_{A}=1, \varepsilon=2, s_{A}=0.15$, except for: (A) $s_{P}=0.05, d_{P}=$

$701 \quad 0.75, d_{A}=1.5 ;(\mathbf{B}) b_{P}=1.2, d_{P}=0.76, a=0.31, d_{A}=0.7 ;(\mathbf{C}) s_{P}=0.14, d_{P}=0.4, a=$

$7020.9, s_{A}=0.115, d_{A}=2.1$; (D) $b_{P}=1.5, s_{P} 0.61, d_{P}=0.67, a=2, h=0.01, \varepsilon=1, s_{A}=$

$7032, d_{A}=1.1 ;(\mathbf{E}) d_{P}=0.2, d_{A}=1.5 ;(\mathbf{F}) b_{P}=1.5, s_{P}=0.6, d_{P}=0.7, a=1.95, h=$

$0.01, \varepsilon=1, s_{A}=2, d_{A}=0.9 ;(\mathbf{G}) d_{P}=0.2, \varepsilon=1, d_{A}=0.7$. 
Facultative

Plants /

Obligate

Animals

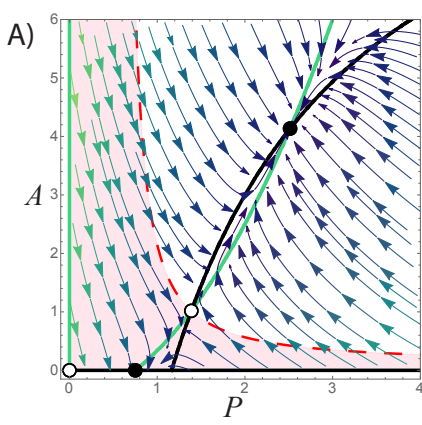

Facultative Plants / Facultative Animals
- Stable
- Saddle
- Unstable
$\sim$ Plant Nullcline
Animal Nullcline
- Separatrix

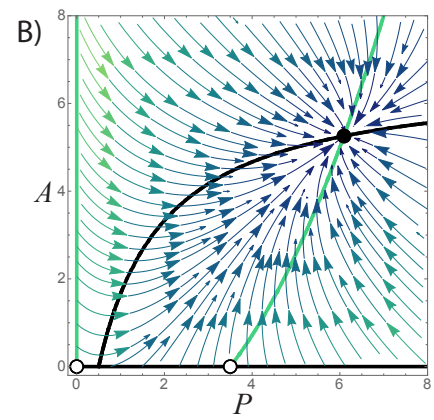

C)

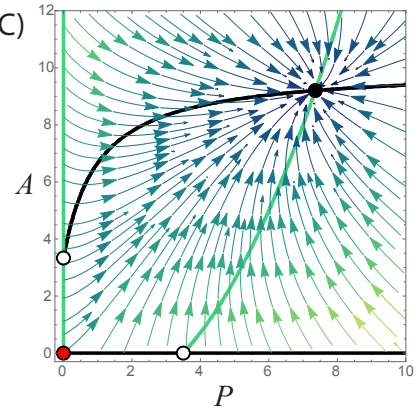

Figure 2. Phase plane diagrams for plant seed-disperser mutualisms, where animals

\section{7}

708

709

710

711 terminology follow Fig. 1. When animals are obligate mutualists (A), both partners may

712 experience threshold effects. Otherwise, both partners experience growth from low density

$713(\mathbf{B}, \mathbf{C})$, resulting in a single stable coexistence equilibrium. Parameter values are fixed to

714 the following $b_{P}=1, f=1, g=1, s_{P}=0.2, \sigma=0.2, d_{P}=0.3, a=1, h=1, b_{A}=$

$7151, s_{A}=0.15$, except for: (A) $d_{P}=0.85, a=2, h=0.5, b_{A}=1, \varepsilon=0.93, s_{A}=0.08, d_{A}=$ 716

$2 ;(\mathbf{B}) \varepsilon=1.5, d_{A}=1.5 ;(\mathbf{C}) \varepsilon=1, d_{A}=0.5$. 

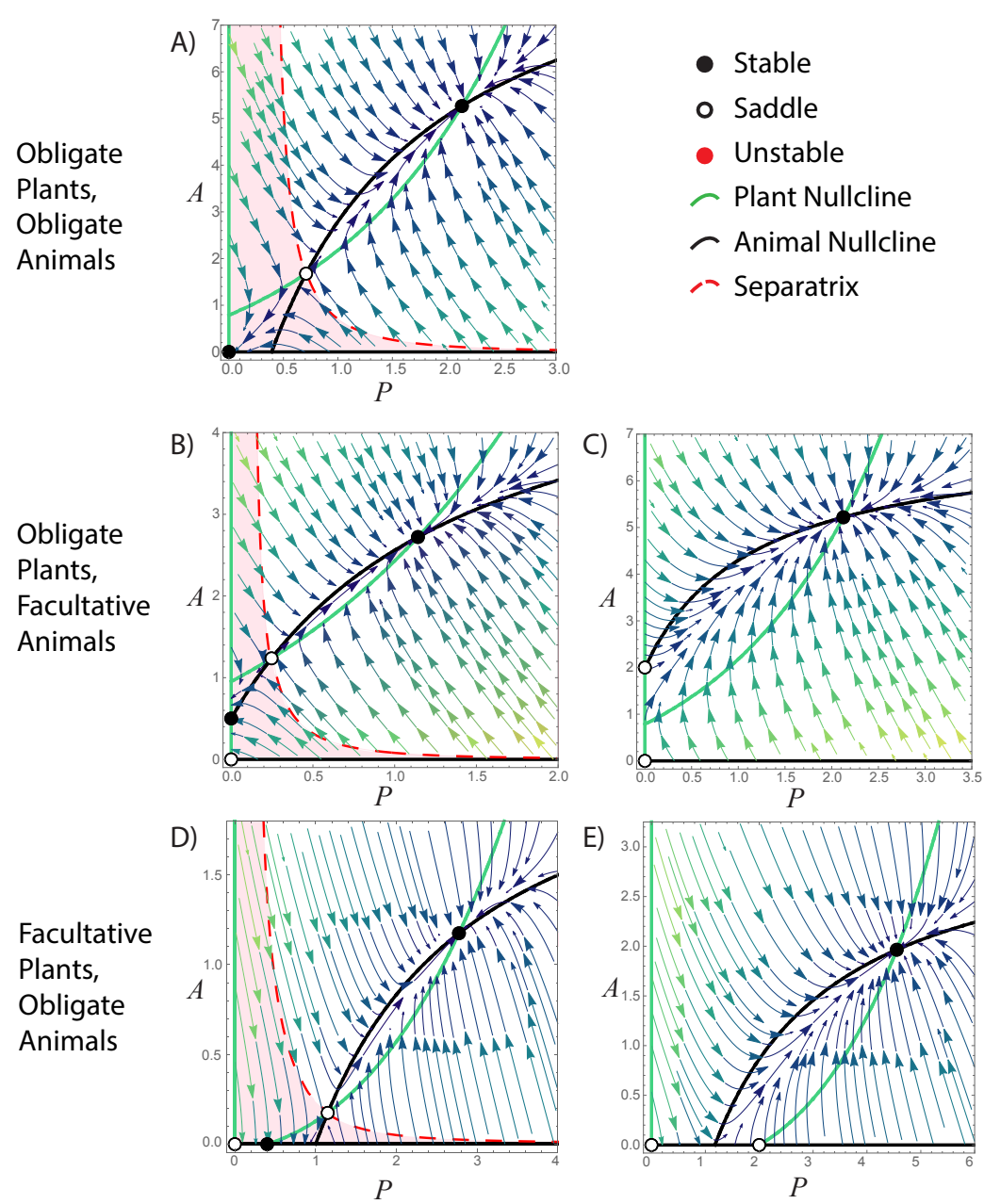

Facultative

Plants,

Facultative

Animals

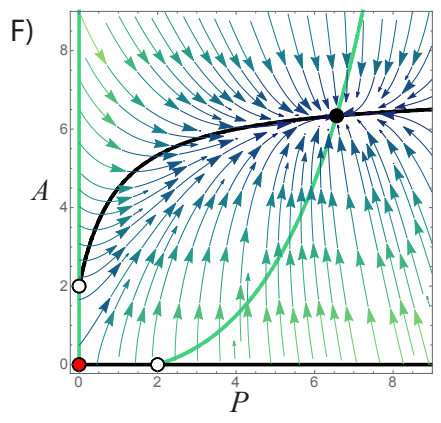

718 Figure 3. Phase plane diagrams for plant seed-disperser mutualisms, where animals

719 increase seed germination in plants. Formatting and terminology follow Fig. 1. When

720 either partner is an obligate mutualist, both partners may experience threshold effects (A, B, 
721 D). When either partner is a facultative mutualist, the system may instead exhibit a single

722 coexistence equilibrium with growth from low density for both partners $(\mathbf{C}, \mathbf{E}, \mathbf{F})$.

723 Parameter values are fixed to the following $b_{P}=1, f=1, g=0.5, \gamma=0.5, s_{P}=$

$7240.05, d_{P}=0.7, a=0.85, h=1, b_{A}=1, \varepsilon=1, s_{A}=0.2, d_{A}=1.5$, except for: $(\mathbf{A}) \varepsilon=$

$7252, s_{A}=0.15 ;(\mathbf{B}) s_{A}=0.7, d_{A}=0.9 ;(\mathbf{C}) d_{A}=0.6 ;(\mathbf{D}) d_{P}=0.48, a=1 ;(\mathbf{E}) d_{P}=$

$7260.4, s_{A}=0.15 ;(\mathbf{F}) a=1, d_{A}=0.6$. 

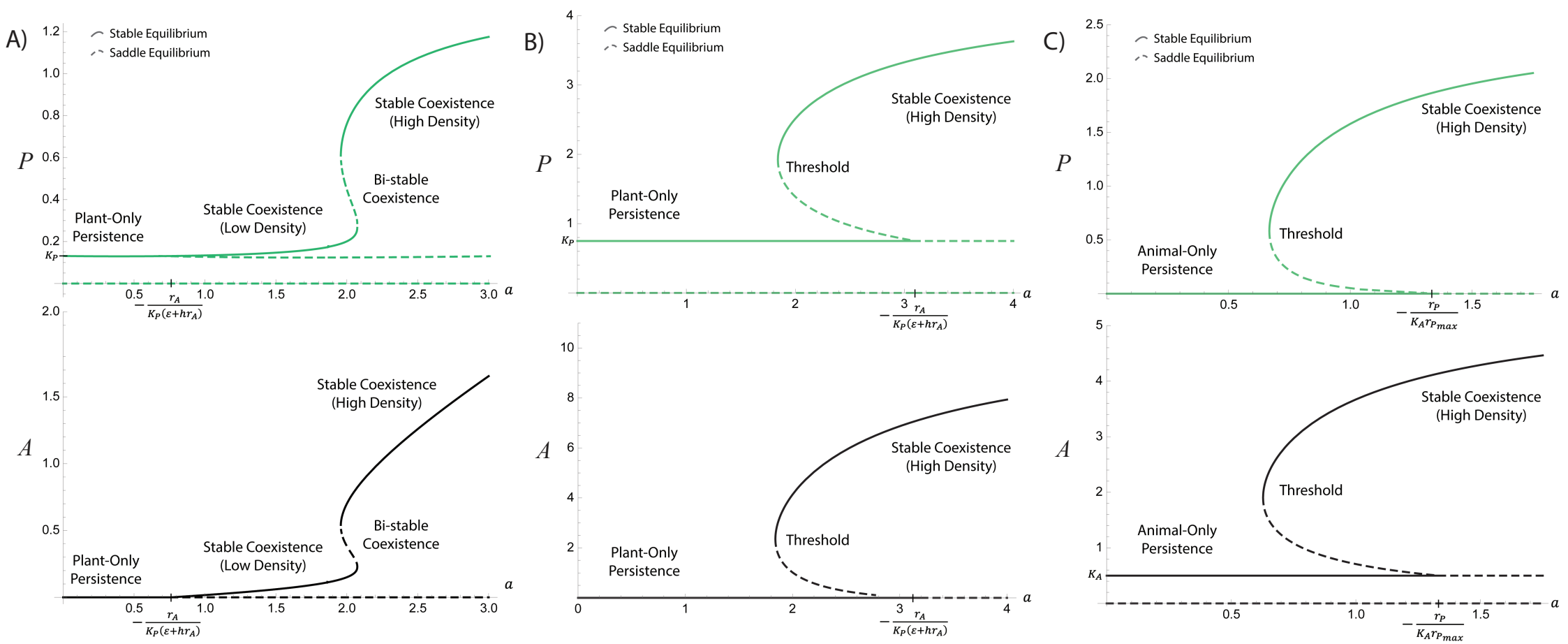

728 Figure 4. Bifurcation diagrams: animal foraging efficiency (a) mediates the dynamics of pollination and seed dispersal

729 mutualisms. Green (top) and black (bottom) curves show plant and animal equilibrium density, respectively, across a range of animal

730 foraging efficiencies or attack rate on plants' rewards ( $a$, x-axis). This parameter mediates both animal benefits from plants (foraging

731 on rewards) and plant benefits from animals (foraging rate determines the rate at which plant gametes are transported). When multiple 
curves occur at the same $a$ value, multiple equilibria are possible. Assuming feasible coexistence: (A) Pollination mutualisms with

733 facultative plants: At low $a$, stable coexistence occurs at low density for both partners, whereas at high $a$, stable coexistence occurs at

734 high density. At intermediate $a$, coexistence occurs at either low or high density (bistable coexistence) depending on the initial

735 densities of the mutualists (whether densities are below or above the separatrix in Fig. 1D, 1F). (B) Seed dispersal with obligate animal

736 mutualists that reduce plant negative density-dependence: At low $a$, stable coexistence occurs if populations are initially at high

737 density. Below a threshold in initial density (separatrix in Fig. 2A), the mutualism collapses and animals go extinct. As $a$ increases,

738 this threshold becomes lower and lower until populations beginning at any positive density exceed it (when $\left.a \geq-\frac{r_{A}}{K_{P}\left(\varepsilon+h r_{A}\right)}\right)$, ensuring

739 stable coexistence. Pollination mutualisms and seed dispersal mutualisms that increase germination also exhibit these dynamics. (C)

740 Seed dispersal mutualisms that increase germination of obligate plants: The transition from threshold effects to stable coexistence

741 occurs when $a \geq-\frac{r_{P}}{K_{A} r_{P_{\max }}}$. Saddle point equilibria are dashed lines, while stable equilibria are solid. In all cases, extinction of both

742 partners and single-species persistence of facultative partners (at $K_{i}$ ) are possible equilibria. Coexistence is infeasible at very low $a$,

743 permitting only single-species persistence or extinction. Note that the inverse of animal attack rate on plants' rewards $(1 / a)$ can be

744 interpreted as animals' search time for plant rewards, where higher search time could be caused by limitations of the animals' sensory

745 ability for identifying available food on the landscape. Attack rate can also be interpreted as animals' preference, where lower 
746 preference could result from lower quality of the provided rewards or risk associated with accessing them. Parameters are fixed to: (A)

$747 b_{P}=1.5, f=0.5, \varphi=0.5, g=1, s_{P}=0.61, d_{P}=0.67, h=0.01, b_{A}=1, \varepsilon=1, s_{A}=0.2, d_{A}=1.1 ;(\mathbf{B}) b_{P}=1, f=1, g=1, s_{P}=$

$7480.2, \sigma=0.2, d_{P}=0.85, h=0.5, b_{A}=1, \varepsilon=0.93, s_{A}=0.08, d_{A}=2 ;(\mathbf{C}) b_{P}=1, f=1, g=0.5, \gamma=0.5, s_{P}=0.05, d_{P}=0.7, h=$

$7491, b_{A}=1, \varepsilon=1, s_{A}=0.2, d_{A}=0.9$, while $a$ is varied. 


\begin{tabular}{|c|c|c|c|c|}
\hline & $\begin{array}{c}\text { Animal foraging } \\
\text { efficiency }(a)\end{array}$ & $\begin{array}{l}\text { Both obligate } \\
\left(r_{i} \leq 0\right)\end{array}$ & $\begin{array}{l}\text { One obligate, } \\
\text { one facultative }\end{array}$ & $\begin{array}{l}\text { Both facultative } \\
\quad\left(r_{i}>0\right)\end{array}$ \\
\hline \multirow{4}{*}{$\begin{array}{l}\text { Stressful } \\
\text { environment } \\
\quad\left(\text { low } K_{i}\right)\end{array}$} & Very low & \multicolumn{2}{|c|}{ Infeasible } & \multirow[b]{2}{*}{ Stable coexistence } \\
\hline & Low & \multirow{3}{*}{$\begin{array}{c}\text { Threshold effects } \\
\text { (Pollination: } \\
\text { Allee effects) }\end{array}$} & Threshold effects & \\
\hline & High & & \multicolumn{2}{|c|}{ (Pollination $r_{A} \approx 0$, low $K_{P}>0$ : Bistable) } \\
\hline & Very high & & Stable coexistence & \\
\hline \multirow{5}{*}{$\begin{array}{c}\text { Benign } \\
\text { environment } \\
\text { (high } K_{i} \text { ) }\end{array}$} & Very low & \multicolumn{2}{|c|}{ Infeasible } & \multirow{5}{*}{ Stable coexistence } \\
\hline & Low & \multirow{4}{*}{$\begin{array}{c}\text { Threshold effects } \\
\text { (Pollination: } \\
\text { Allee effects) }\end{array}$} & \multirow{4}{*}{ Stable coexistence } & \\
\hline & & & & \\
\hline & High & & & \\
\hline & Very high & & & \\
\hline
\end{tabular}

752 Figure 5. Summary diagram of our results: pollination and seed dispersal dynamics under different ecological scenarios.

753 Critical transitions between different dynamics are strongly controlled by both partners' obligacy (governed by per-capita growth rate,

$754 r_{i}$ ), the level of environmental hostility (governed by carrying capacity, $K_{i}=r_{i} / s_{i}$ ), and animals' foraging efficiency on rewards (or

755 attack rate, $a)$. Species may be facultative mutualists $\left(r_{i}>0\right)$ or obligate upon their partners for persistence $\left(r_{i} \leq 0\right)$. In hostile

756 environments, facultative and obligate mutualists are assumed to exhibit low and very negative "carrying capacities," respectively. In

757 benign environments, facultative or obligate species have high or barely negative carrying capacities, respectively. At very low animal

758 foraging efficiency, coexistence is infeasible if at least one species is obligate. When both species are facultative, stable coexistence

759 always occurs when feasible, regardless of obligacy or environmental hostility. When both species are obligate, threshold effects (and 
760 Allee effects for pollination mutualisms) occur when coexistence is feasible. In seed dispersal mutualisms: i) threshold effects occur in 761 hostile environments when at least one species is obligate, unless attack rate is very high in which case stable coexistence may occur, 762 and ii) stable coexistence always occurs in benign environments when at least one partner is facultative. The asterisk indicates that in 763 pollination mutualisms, i) and ii) occur specifically when plants are the facultative partner and animals are obligate. Otherwise, 764 threshold effects and Allee effects occur when animal-pollinated plants are obligate, regardless of animals' obligacy and the 765 environmental condition. Pollination mutualisms uniquely exhibit bistability when plants are facultative and $r_{A}$ is close to zero, 766 regardless of animal obligacy. 
Table 1. Table of parameters.

Parameter

\begin{tabular}{|c|c|c|}
\hline$a$ & Per-plant attack rate on rewards & {$\left[P^{-1} t^{-1}\right]$} \\
\hline$b_{i}$ & Per-capita birth or seed production rate & {$\left[t^{-1}\right]$} \\
\hline$\varepsilon$ & $\begin{array}{c}\text { Efficiency of converting food rewards to animal } \\
\text { offspring }\end{array}$ & Unitless \\
\hline$d_{i}$ & Per-capita death rate & {$\left[t^{-1}\right]$} \\
\hline$f$ & $\begin{array}{l}\text { Fraction of flowers pollinated due to selfing, wind- } \\
\text { pollination, etc., } 0<f \leq 1\end{array}$ & Unitless \\
\hline$\varphi$ & $\begin{array}{l}\text { Fraction of flowers pollinated due to animal- } \\
\text { pollination, } 0<\varphi \leq 1-f\end{array}$ & Unitless \\
\hline$g$ & $\begin{array}{l}\text { Fraction of seeds that germinate in the absence of } \\
\text { animal dispersal, } 0<g \leq 1\end{array}$ & Unitless \\
\hline$\gamma$ & $\begin{array}{l}\text { Fraction of seeds that germinate due to animal } \\
\text { dispersal (improved seed condition), } \\
\qquad 0<\gamma \leq 1-g\end{array}$ & Unitless \\
\hline$h$ & Handling time on rewards & {$[t]$} \\
\hline$s_{i}$ & $\begin{array}{l}\text { Negative density-dependence due to, e.g., self- } \\
\text { limitation during recruitment, Janzen-Connell } \\
\text { effect }\end{array}$ & $\begin{array}{l}S_{P}:\left[P^{-2}\right] \text { or } \\
S_{A}:\left[A^{-2} t^{-1}\right]\end{array}$ \\
\hline$\sigma$ & $\begin{array}{l}\text { Reduced negative density-dependence due to } \\
\text { animal dispersal (movement to safe sites), } \\
\qquad 0<\sigma \leq s_{P}\end{array}$ & {$\left[P^{-2}\right]$} \\
\hline
\end{tabular}

Interpretation

\section{Units}




\section{Appendix A: Mathematical Analysis}

In all our models (Eqns. 3, 6, 7), plant and animal populations have "trivial" nullclines: $\overline{\boldsymbol{P}}=0$, a vertical line along the y-axis, and $\overline{\boldsymbol{A}}=0$, a horizontal line along the x-axis. The intersection of these trivial nullclines results in a trivial extinction equilibrium $(\overline{\boldsymbol{P}}=0, \overline{\boldsymbol{A}}=0)$ in each model. Below, we describe the geometry of the nontrival nullclines (hereafter, simply "nullclines") in the ecologically relevant region of the plane, when $\boldsymbol{P} \geq 0, \boldsymbol{A} \geq 0$ (hereafter, simply "the positive quadrant"). All our models are structurally unstable (Rohr et al. 2014), such that smooth transitions in parameter values shift the nullclines so that they may intersect in various ways or even fail to intersect in the positive quadrant, with different dynamical outcomes for each case (Fig. 4). Below, we also describe how the nullcline geometries are dependent on obligacy to mutualism, which leads to structural instability.

The animal population follows the same dynamics (Eqn. 1) and thus has the same nontrivial nullcline in all our models (Figs. 1-3, black):

$$
\overline{\boldsymbol{A}}=\frac{r_{A}}{s_{A}}+\frac{\varepsilon}{s_{A}} \frac{a \boldsymbol{P}}{(1+a h \boldsymbol{P})}
$$

where $r_{A}=b_{A}-d_{A}$. This equation describes the balance between negative density-dependence, benefit from mutualism, and intrinsic growth or decay for facultative $\left(r_{A}>0\right)$ or obligate $\left(r_{A} \leq\right.$ 0) animal populations, respectively. When $\boldsymbol{A}>\overline{\boldsymbol{A}}$, negative density-dependence is stronger than benefit from mutualism and/or intrinsic growth, causing $\boldsymbol{A}$ to decrease. When $\boldsymbol{A}<\overline{\boldsymbol{A}}$, negative density-dependence is weaker and $\boldsymbol{A}$ increases.

Specifically, Eqn. A1 is a concave down, increasing function that saturates with respect to plant density, $\boldsymbol{P}$. The benefit from mutualism can be isolated as $\frac{\varepsilon}{s_{A}} \frac{a \boldsymbol{P}}{(1+a h \boldsymbol{P})}$; it is the density 
above carrying capacity $\left(K_{A}=r_{A} / s_{A}\right)$ that the animal population achieves by foraging on plant

798 from handling food rewards $(h)$. This sets an upper bound (horizontal asymptote) on animal

799 density at $\overline{\boldsymbol{A}}=K_{A}+\frac{\varepsilon}{s_{A} h}$. When facultative, the animal nullcline intersects the y-axis in the

800 positive quadrant at animal carrying capacity $\overline{\boldsymbol{A}}_{0, y}=K_{A}$. All else being equal, decreasing $r_{A}$

801 pushes the visible part of the animal nullcline down so that when the animal population becomes

802 obligate, its nullcline intersects the y-axis at zero or negative values (not shown). Instead, for

803 obligate animals the $\mathrm{x}$-intercept becomes visible in the positive quadrant at

$$
\overline{\boldsymbol{A}}_{x, 0}=\frac{-r_{A}}{a\left(\varepsilon+h r_{A}\right)} \text {. }
$$
pollinated plants (Fig. 1, green) is:

$$
\boldsymbol{A}=\frac{\left(r_{P}-s_{P} \overline{\boldsymbol{P}}\right)(1+a h \overline{\boldsymbol{P}})}{a \overline{\boldsymbol{P}}\left(s_{P} \overline{\boldsymbol{P}}-r_{P_{\max }}\right)}
$$

$811 \boldsymbol{P}=r_{P_{\max }} / s_{P}$, where $\boldsymbol{P}=r_{P_{\max }} / s_{P}$ represents the plant's maximum population density. When

812 plants are obligate $\left(r_{P} \leq 0\right.$, Fig. 1A-B), the nullcline is a U-shape. All else being equal,

813 increasing $r_{P}$ pushes the minimum point of the $\mathrm{U}$ down towards the x-axis, so that when the plant 814 population becomes facultative, it intersects, flipping into a cubic shape. Therefore, when plants

815 are facultative $\left(r_{P}>0\right.$, Fig. 1C-G), the nullcline is concave up at high density but concave down 816 at low density, though this inflection may not be visible in the positive quadrant. The $\mathrm{x}$-intercept 
817 is the plant carrying capacity $\overline{\boldsymbol{P}}_{x, 0}=\frac{r_{P}}{s_{P}}=K_{P}>0$. Inside the $\mathrm{U}$ or to the left of the plant

818 nullcline, plant density increases; to the right or under, plant density decreases due to strong

819 negative density-dependence.

820 The equation for the nullcline of animal-dispersed plants that benefit through reduced negative density-dependence (Fig. 2, green) is:

$$
\boldsymbol{A}=\frac{\left(r_{P}-s_{P} \overline{\boldsymbol{P}}\right)(1+a h \overline{\boldsymbol{P}})}{a\left(\left(s_{P}-\sigma\right) \overline{\boldsymbol{P}}-r_{P}\right)}
$$

823 In the positive quadrant, the nullcline is increasing, concave up and saturates to a maximum plant

824 density of $\boldsymbol{P}=r_{P} /\left(s_{P}-\sigma\right)$ when $s_{P}>\sigma$. This maximum disappears to infinity when $s_{P}=\sigma$,

825 that is, when dispersers can completely remove sources of negative density-dependence. Thus,

826 based on the nullcline alone, benefits to plants would increase indefinitely with increasing animal

827 density. However, animal visitation rate saturates due to handling time (encoded in the Holling

828 type II functional response), bounding the benefits to plants by intersecting the plant nullcline at

829 high density. Because we consider only facultative plants for this mutualism, the nullcline

830 always has an x-intercept at plant carrying capacity $\bar{P}_{x, 0}=K_{P}>0$ but no y-intercept in the

831 positive quadrant.

833 (Fig. 3, green) is:

$$
\boldsymbol{A}=\frac{\left(r_{P}-s_{P} \overline{\boldsymbol{P}}\right)(1+a h \overline{\boldsymbol{P}})}{a\left(s_{P} \overline{\boldsymbol{P}}-r_{P_{\max }}\right)}
$$

835 where $r_{P_{\max }}=r_{P}+b_{P} f \gamma$ is the maximum per-capita growth rate of the plant population in the

836 presence of dispersers. For feasible coexistence, $r_{P_{\max }}>0$. In the positive quadrant, the nullcline 
837 is an increasing, concave up curve, bounded by a vertical asymptote at $\boldsymbol{P}=r_{P_{\max }} / s_{P}$, the

838 maximum plant density in the presence of mutualism. The nullcline intersects the $\mathrm{x}$-axis at plant

839 carrying capacity $\overline{\boldsymbol{P}}_{x, 0}=\frac{r_{P}}{s_{P}}=K_{P}$ which is visible in the positive quadrant when plants are

840 facultative $\left(r_{P}>0\right)$. It intersects the y-axis at

$$
\overline{\boldsymbol{P}}_{0, y}=\frac{-r_{P}}{\operatorname{ar}_{P_{\max }}}
$$

842 which is visible in positive quadrant when plants are obligate $\left(r_{P} \leq 0\right)$.

\section{Note on Functional Forms}

847 dispersal services $\left(\mathcal{S}_{D}\right)$ are assumed to be equal to the per-plant consumption rate. Reproductive

848 services are assumed to saturate according to $\mathcal{S} /(\kappa+\mathcal{S})$, where $\kappa$ is saturation coefficient for

849 benefit from animal visitation rate with units $\left[A t^{-1}\right]$ or $\left[A P^{-1} t^{-1}\right]$ for pollination or seed

850 dispersal mutualisms, respectively. For convenience, we set $\kappa=1$ in the main text.

\section{References}

852 Rohr, R.P., Saavedra, S. \& Bascompte, J. (2014). On the structural stability of mutualistic 